\title{
Clinical Profile of Patients Undergoing Temporary Transvenous Pacing in a Tertiary Cardiac Care Centre in Nepal
}

\author{
Manju Sharma, Sunil C Jha, Arun Sayami, Ratna M Gajurel, Chandra M Poudel, Rajaram Khanal, \\ Surya R Pathak, Shovit Thapa, Suman Adhikari, Vijay Yadav \\ Department of Cardiology, Maharajgunj Medical Campus, Manmohan Cardiothoracic Vascular and Transplant \\ Center, Institute of Medicine, Maharajgunj, Kathmandu, Nepal
}

\section{Corresponding author:}

\section{Manju Sharma, MBBS, MD}

Department of Cardiology, Maharajgunj Medical Campus, Manmohan Cardiothoracic Vascular and Transplant Center, Institute of Medicine, Maharajgunj, Kathmandu, Nepal

Email:man_nju@hotmail.com

Submitted : Jun 6, 2020

Accepted : Jul 28, 2020

\begin{abstract}
\section{Introduction}

Temporary transvenous pacemaker insertion is an emergency lifesaving procedure for patients with hemodynamically unstable and life-threatening bradyarrythmias. The aim of this study was to analyze demographics, indications, route of insertion and complications in patients undergoing temporary transvenous pacemaker implantation.
\end{abstract}

\section{Methods}

This was a retrospective observational study conducted at a tertiary-care center in Nepal. The hospital records of patients who had undergone temporary transvenous pacemaker implantation between July 2015 and June 2019 were reviewed.

\section{Results}

A total of 343 patients with mean age of $65.52 \pm 16.09$ years received temporary transvenous pacing. Out of these 205 (59.8\%) were males. Greater proportion of patients were between the age group of 70-80 years $(n=76,22.2 \%)$. Hypertension ( $n=97,28.3 \%$ ) was the most common comorbidity noted. The most common indication for temporary pacing was symptomatic complete heart block 165 (59.6\%). Total of 288 (84\%) patients received permanent pacemakers while 55(16\%) had reversible cause so TPI was removed. Right Femoral vein was the most common (99\%) venous access site. Among the 343 patients, complications were observed in $29(8.4 \%)$ of cases during and after the temporary transvenous pacemaker insertion. The overall mortality stood low at $2 \%(n=7)$.

\section{Conclusion}

Temporary transvenous pacemaker insertion is required in elderly population presenting with bradyarrythmias and occasionally in acute myocardial infarction presenting with bradyarrythmias as complication. Temporary pacemaker insertion was overall a safe procedure with infrequent serious complications; however, strategies to avoid and alleviate such complications (RV perforation) should be sought and implemented.

Keywords: Complete heart block, myocardial infarction, temporary transvenous pacemaker 


\section{INTRODUCTION}

$\mathrm{T}$ emporary transvenous pacing (TTP) involves electrical cardiac stimulation to treat bradyarrythmias or tachyarrhythmia until it resolves or until long-term therapy can be initiated. ${ }^{1}$ The purpose of temporary pacing is to re- establish circulatory integrity and normal hemodynamics that are acutely compromised by a slow or fast heart rate. ${ }^{2}$ The indications for temporary pacemaker insertion with its complications are well documented. Awareness of the potential complications allows quicker diagnosis and may therefore be lifesaving. ${ }^{3,4}$

Temporary transvenous endocardial pacing was first described by Furman and Robinson in $1958 .{ }^{5}$ Although there are several types of temporary cardiac pacing like transvenous, transcutaneous, transesophageal, epicardial, the most widely used is transvenous, i.e., peripheral venous puncture and placement of an electrode in the right cardiac chamber. ${ }^{6}$ This type of pacing has been employed since the 1960s, when it was first used in patients with permanent arrhythmias. ${ }^{7}$ Since that time, it has become common in the majority of hospitals, and currently there are well-established indications for its use. ${ }^{8}$

TTP services are in place in the country for more than a decade now. But to date, there have not been large scale studies on this topic. The objective of this study is to analyze the clinical profile, indications and complications of temporary transvenous pacing in Nepali population presenting to Manmohan Cardiothoracic Vascular and Transplant Centre (MCVTC).

\section{METHODS}

This was hospital based, retrospective observational study conducted at Department of Cardiology, Manmohan Cardiothoracic Vascular and Transplant Centre, Maharajgunj, Kathmandu. All patients who underwent temporary transvenous pacemaker insertion between July 2015 and June 2019 were included and their hospital records analysed. Ethical approval was obtained from the Institutional Review Committee of Institute of Medicine. From the records, data were obtained on age, sex, diagnosis, indications of temporary pacemaker insertion, route of access, duration, need for a permanent pacemaker, and complications during hospital stay.

The pacemaker was placed in a cardiac catheterization laboratory under fluoroscopy guidance. The procedure was performed under local anesthesia. The right femoral vein was used in most of the patients except in few cases where right jugular vein was used. Bipolar electrode catheters (caliber, $6 \mathrm{Fr}$ ) were inserted using the
Seldinger technique and positioned in the right ventricular apex under fluoroscopy guidance; with a pacing threshold of 0.5-0.1 V considered adequate. Temporary pacing was initially set at voltage values twice the threshold level. In all cases, chest X-rays were obtained to check correct positioning of the electrode, together with an electrocardiogram showing in all a left bundle branch block after implantation. Echocardiographic assessment was also done to confirm lead position in right ventricle (RV) and rule out pericardial effusion. After placing the pacemaker, most of the patients were given lowmolecular-weight heparin at prophylactic doses. The patients were advised limb immobilization along with continuous electrocardiographic monitoring until a permanent pacemaker was implanted or indication for a temporary pacemaker ceased to exist.

Data were entered in Excel and exported to SPSS 21.0 for further analysis. Descriptive statistics such as mean for continuous variables and percentages, ratios, etc. for categorical variables were computed. The association between two categorical variables were assessed through the use of Chi-square test. Independent t-test used to compare mean age of two groups, myocardial infarction (MI) and nonmyocardial infarction (non-MI). Fisher's exact test was used for bivariate analysis where number of observations were below five in any of the category. The results were considered statistically significant if $p<0.05$.

\section{RESULTS}

A total of 343 patients underwent temporary transvenous pacemaker insertion at MCVTC between July 2015 and June 2019. Out of these 205 (59.8\%) were male and 138 (40.23\%) were

Table 1. Clinical variables of the study population

\begin{tabular}{lc}
\hline \multicolumn{1}{c}{ Clinical Characteristics } & Values \\
\hline Age (years) & $65.52 \pm 16.09$ \\
Male & $205(59.8 \%)$ \\
Hypertension & $97(28.3 \%)$ \\
Diabetes & $33(9.6 \%)$ \\
lschemic heart disease & $24(7.0 \%)$ \\
Chronic obstructive pulmonary & \\
$\quad$ diseases & $23(6.7 \%)$ \\
Cardiac surgery & $19(5.5 \%)$ \\
Degenerative valvular heart & \\
$\quad$ disease & $17(5.0 \%)$ \\
Chronic kidney disease & $16(4.7 \%)$ \\
History of stroke & $13(3.8 \%)$ \\
Acute kidney injury & $13(3.8 \%)$ \\
Dilated cardiomyopathy & $12(3.5 \%)$ \\
Rheumatic heart disease & $11(3.2 \%)$ \\
Seizure & $4(1.2 \%)$ \\
Malignant disease & $3(0.9 \%)$ \\
\hline
\end{tabular}




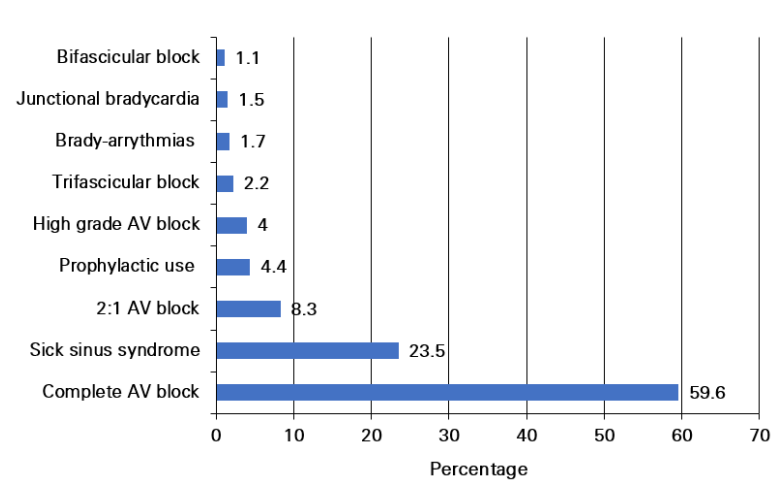

Fig 1. Indications for temporary transvenous pacemaker insertion

female with male to female ratio of 1.48 . The mean age $( \pm S D)$ was $65.52 \pm 16.09$ years (Table 1$)$. The minimum age was 8 years and maximum was 99 years with $3(0.9 \%)$ cases $<20$ years of age, almost $136(39.7 \%)$ cases aged $>60$ years and $51(14.9 \%)$ cases aged $>80$ years. A significant proportion (76, $22.2 \%$ ) of patients were in the seventh decade of life.

A total of $285(83.2 \%)$ cases had one or more comorbidities. Hypertension was found to be most common comorbidity present among 97 (28.3\%) cases, followed by diabetes mellitus 33(9.6\%), Among the participants $24(7.0 \%)$ had history of ischemic heart disease, 23 (6.7\%) had chronic obstructive pulmonary disease (COPD), 17 (5.0\%) had degenerative valvular heart disease, 19 (5.5\%) were in post cardiac surgery status, $16(4.7 \%)$ had chronic kidney disease, 13 (3.8\%) had history of stroke, 13 (3.8\%) had acute kidney injury, 12 (3.5\%) of the cases had dilated cardiomyopathy. Similarly, $11(3.2 \%)$ cases had rheumatic heart diseases, 4 $(1.2 \%)$ cases had experienced seizures and $3(0.9 \%)$ of the cases had malignant diseases (Table 1).

The most common indication for temporary transvenous pacemaker insertion (TPI) was complete atrioventricular block 165 (59.6\%) followed by sick sinus syndrome 65 (23.5\%), 2:1 AV block 23 (8.3\%), high-grade AV block 11 (4.0\%), Trifasicular block 6 (2.2\%), Junctional Bradycardia 4 $(1.5 \%)$ and Bifasicular block $3(1.1 \%)$, prophylactic use due to generator replacement in 15 patients $(4.4 \%)$, brady-arrythmias due to drug intoxication or dyselectrolytemia in $6(1.7 \%)$ patients (Figure1).

Total of $288(84 \%)$ patients received permanent pacemakers while $55(16 \%)$ had reversible cause so TPI was removed. Among the 55 patients, 49 patients $(14.3 \%)$ presented with acute myocardial infarction (AMI) complicated by some advanced type of conduction disorder such as complete heart block (CHB), 2:1 atrioventricular (AV) Block, High degree AV block, junctional bradycardia and sinus bradycardia in one patient. Among acute MI patients, conduction disorder was higher among acute inferior wall myocardial infarction (IVMI) $7.9 \%$ patients than acute anterior wall myocardial infarction (AWMI) 5.2\%. There were $4(1.2 \%)$ patients with non-ST segment elevetion myocardial infarction (NSTEMI). This group of patients did not show specific difference in comorbidities when compared to the group without MI except for acute kidney injury (Table 2).

The mean ventricular rate at the time of temporary transvenous pacemaker placement was $38 \pm 6 \mathrm{bpm}$. The duration of temporary transvenous pacing was 3.4 days (range, 1-10 days). Implantation was through the femoral vein in $99 \%$ of the cases, with the right femoral vein. The right internal jugular vein was used in few cases who presented in respiratory distress.

Complications were infrequent and usually minor. A total of $29(8.4 \%)$ cases experienced some form of complications during and after the temporary transvenous pacemaker implantation.

Complicated access like minor bleed access site, multiple punctures, and arterial puncture was noted

Table 2. Comparison of clinical characteristics of the groups with and without acute myocardial infarction

\begin{tabular}{|c|c|c|c|}
\hline Clinical Characteristics & $\begin{array}{c}\text { MI Group } \\
n(\%)=49(14.3 \%)\end{array}$ & $\begin{array}{c}\text { Non-MI group } \\
n(\%)=294(85.7 \%)\end{array}$ & $\mathrm{p}$-value \\
\hline Age & $61.78 \pm 14.90$ & $66.15 \pm 16.22$ & $0.08^{\#}$ \\
\hline Gender (Male) & $34(16.6)$ & $171(83.4)$ & 0.14 \\
\hline Hypertension (HTN) & $14(14.4)$ & $83(85.6)$ & 0.9 \\
\hline Diabetes & $4(12.1)$ & $29(87.9)$ & $1.0^{*}$ \\
\hline History of stroke & $2(15.4)$ & $11(84.6)$ & $1.0^{*}$ \\
\hline Chronic kidney disease & $1(6.3)$ & 15(93.8) & $0.49 *$ \\
\hline Chronic obstructive pulmonary diseases (COPD) & $1(4.3)$ & 22(95.7) & $0.22 *$ \\
\hline Dilated cardiomyopathy (DCM) & 0 & $12(100)$ & $0.23^{*}$ \\
\hline Rheumatic heart disease (RHD) & 0 & $11(100)$ & $0.38 *$ \\
\hline Degenerative valvular heart disease (DVHD) & 0 & $17(100)$ & $0.15^{*}$ \\
\hline Acute kidney injury (AKI) & $6(46.2)$ & $7(53.8)$ & 0.005 \\
\hline Seizure & 0 & $4(100)$ & $1.00 *$ \\
\hline
\end{tabular}


in 11 patients. System malfunctions (problems with connections and lead placement or inappropriate setup of the device) was noted in five cases. Two cases developed ventricular tachycardia/ ventricular fibrillation (VTNF) during temporary transvenous pacemaker placement in our study. Procedurerelated ventricular tachycardia/ventricular fibrillation were not included as serious complications as these usually arise during device insertion and can be corrected at this stage. There were no cases of infection at the puncture site, deep venous thrombosis or pneumothorax.

The overall mortality stood low at $2 \%(n=7)$ in our study. Among these patients, six patients were diagnosed case of AMl and one was non MI patient. Among these six patients, one died as a result of cardiac tamponade secondary to cardiac perforation after removing the pacemaker lead, other five deaths resulted due to other complications of MI. One non Ml patient died due to RV perforation attributable to stiff temporary pacemaker lead.

Right ventricle (RV) perforation occurred in six $(1.7 \%)$ cases. Two patients underwent RV repair. One patient simultaneously underwent epicardial pacemaker implantation following RV repair. One died post-surgery and one died during resuscitation while other was discharged. Cardiac tamponade was noted in three patients caused by RV perforation with the electrode catheter, all underwent pericardiocentesis, and one underwent permanent pacemaker insertion post recovery, while other was kept in observation. After pericardial effusion was decreasing and patient's condition was improving, pigtail was removed and patient was discharged. Despite pericardiocentesis one died attributable to complication of MI.

\section{DISCUSSION}

This study was conducted to evaluate the demographic details, indications and complications in patients undergoing temporary transvenous pacemaker insertion either as an emergency procedure or electively before permanent pacemaker insertion. A large proportion of patients receiving pacemakers were elderly and it has been well demonstrated in other studies., 2,10 This might probably be due to incidence of degenerative disease as well as ischemic heart disease in this age group of patients. In younger age group below 20 years, Temporary transvenous pacemaker were inserted in patients with congenital or post-surgical complete heart block.

Our study shows that among patients receiving temporary transvenous pacing, male predominance was seen (59.8\% study patients were male). This is comparable to age distribution reported worldwide. ${ }^{11,12}$ The reason for fewer incidence of female cases might be less health seeking behavior of female and less investment of society in female. Also, greater use of TPI in male patients might be due to increased incidence of co-morbidities such as hypertension, diabetes mellitus and ischemic heart disease compared to female.

Elderly patients with AV block or sick sinus syndrome are treated with pacemaker implantation. In our study complete heart block predominated $(51 \%)$, together with symptomatic sinus node disease $(19 \%)$. These results are comparable to that reported in earlier studies.9,13,14 The indications of TPI in our study are very similar to those reported by other authors such as Murphy et al., ${ }^{15}$ who found the main indications to be complete heart block. Second cause being sinus node disease in our study for temporary transvenous pacemaker insertion. This can possibly be explained by the more widespread availability of $24 \mathrm{hr}$ Holter recording that has improved the pick-up rate of sinus node disease in the general population. Also, in previous decades greater number of patients requiring a temporary pacemaker presented with AMI.16,17 In our study only 49 patients presented with AMI with conduction disorders. This change in the proportion of indications is probably due to advances in the treatment (such as revascularization strategies with thrombolysis and angioplasty) of patients with AMI. Conduction disorder was more common among Inferior wall MI patients which was similar to other studies. ${ }^{18}$ Right coronary artery occlusion is usually involved as it vascularizes the AV nodal artery.

The femoral approach, which allows rapid access and easy compression in case of bleeding, ${ }^{12}$ was preferred in our hospital Femoral placement may be accompanied by the least stable wire position and may restrict the patient's mobility by requiring a horizontal position. The other approach via right jugular vein, used in very few cases in our study, was alternative option when patient presented in respiratory distress. Other studies showed a preference to right jugular vein approach concluding fewer complications with fewest infections. ${ }^{19,5}$

There were no remarkable complications during the implantation procedure in this study. In our study, only $8.4 \%$ of the patients presented complications of some kind. This is similar to other reports done worldwide. ${ }^{20}$ Despite the urgency of the procedure, with trained operators and strict sterile techniques, there appears to have no infection at the puncture site noted in our study. In our study, however, the duration of pacing was also shorter than that of other studies probably leading to fewer complications. ${ }^{5}$ Mortality in our study are similar to or lower than those in other series. ${ }^{21}$ Mortality occurred in seven patients (2\%) in our study with six patients presented with AMI and one without AMI. The cause of mortality in AMI patients was contributed to the complications of infarction in all cases as 
majority had presented with cardiogenic shock. The prognosis of intraventricular conduction defects is probably related more to the extent of myocardial injury than to the direct consequences of the block itself. One mortality in non-MI patient occurred due to right ventricular perforation leading to cardiac tamponade. Cardiac perforation with tamponade is one of the most feared complications of intracardiac procedures. The temporary pacing lead is relatively stiff, it is not unusual for these leads to penetrate and occasionally perforate the right ventricular wall. The majority of perforations occur through the right ventricular apex, primarily because this remains a common site of deployment of the ventricular lead, and the myocardial wall is thinner here than at other common pacing sites such as the septum and right ventricular outflow tract. ${ }^{22}$

The limitation of our study is that we collected data retrospectively from hospital records. The data obtained from this single hospital may not be applicable to all patients with temporary Transvenous pacing.

\section{CONCLUSION}

Temporary transvenous pacemaker insertion is a lifesaving procedure as a bridge to permanent pacemaker insertion or recovery. Greater proportion of patients receiving TPI are elderly with complete AV block and occasionally in acute myocardial infarction presenting with bradyarrythmias as complication. Temporary pacemaker insertion was overall a safe procedure with infrequent serious complications; however, strategies to avoid and alleviate such complications should be sought and implemented.

\section{CONFLICT OF INTEREST}

None declared.

\section{ACKNOWLEDGEMENTS}

We are grateful to Dr. Lila Bahadur Basnet, for his assistance with the statistical analysis of this study. We would also like to thank Cardiac Catheter Laboratory and Medical Records Department of MCVTC for their assistance.

\section{REFERENCES}

1. Gregoratos G. Indications and recommendations for pacemaker therapy. Am Fam Physician. 2005 Apr 15;71(8):1563-70

2. López A, Villuendas S, García C, et al. Temporary Pacemakers: Current Use and Complications. Rev Esp Cardiol.2004 Nov:57(11):1045-52.

3. Shah H, Mehmood M, Salahudin M, Altaf A. Risk Factor for Cardiac Permanent Pacemaker Infection. J Nepal Health Res Counc.2019 Aug 4;17(2):158-162.

4. Gammage MD. Temporary cardiac pacing. Heart.2000 Jun;83(6):715-20
5. Yaqub Y, Perez-Verdia A, Jenkins $L A$, et al. Temporary Transvenous Cardiac Pacing in Patients With Acute Myocardial Infarction Predicts Increased Mortality. Cardiol Res.2012 Feb;3(1):1-7

6. Kossaify A. Temporary Endocavitary Pacemakers and their Use and Misuse: the Least is Better. Clin Med Insights Cardiol.2014 Jan; 8: 9-11.

7. Betts TR. Regional survey of temporary transvenous pacing procedures and complications. Postgrad Med J.2003 Aug;79(934):463-5.

8. Kusumoto FM, Schoenfeld MH, Barrett C, et al. 2018 ACC/AHA/ HRS Guideline on the Evaluation and Management of Patients With Bradycardia and Cardiac Conduction Delay: A Report of the American College of Cardiology/American Heart Association Task Force on Clinical Practice Guidelines and the Heart Rhythm Society. Heart Rhythm.2019 Sep;16(9):128-226

9. Khanal J, Poudyal RR, Devkota S,et al. Clinical Profile and Early Complications after Single and Dual Chamber Permanent Pacemaker Implantation at Manmohan Cardiothoracic Vascular and Transplant Centre, Kathmandu, J Nepal Health Res Counc.2015 May-Aug;13(30):138-43.

10. Armaganijan LV, Toff WD, Nielsen JC, et al. Are elderly patients at increased risk of complications following pacemaker implantation? A meta-analysis of randomized trials. Pacing Clin Electrophysiol.2012 Feb;35(2):131-4.

11. Bhat S, Kumar D, Parimoo A. Characteristics, indications and complications in patients undergoing permanent pacemaker implantation: a single centre study. Int J Res Med Sci.2018 Dec;6(12):4053-4057

12. Muñoz J, Prieto MA, Macías I, et al. Efficacy and safety of nonpermanent transvenous pacemaker implantation in an intensive care unit. Med Intensiva .2011 Oct;35(7):410-6

13. Shenthar J, Bohra S, Jetley V, et al. A survey of cardiac implantable electronic device implantation in India: By Indian Society of Electrocardiology and Indian Heart Rhythm Society. Indian Heart J.Jan-Feb 2016;68(1):68-71

14. Muller C, Cernin J, Glogar D, et al. Survival rate and causes of death in patients with pacemakers: dependence on symptoms leading to pacemaker implantation. Eur Heart J.1988 Sep;9(9):1003-9

15. Murphy JJ. Problems with temporary cardiac pacing. Expecting trainees in medicine to perform transvenous pacing is no longer acceptable. BMJ.2001 Sep 8;323(7312):527

16. Rosenfeld LE. Bradyarrhythmias, abnormalities of conduction, and indications for pacing in acute myocardial infarction. Cardiol Clin.1988 Feb;6(1):49-61.

17. Berger $P B$, Ruocco NA, Ryan TJ,et al . Incidence and prognostic implications of heart block complicating inferior myocardial infarction treated with thrombolytic therapy: results from TIMI II. J Am Coll Cardiol.1992 Sep;20(3):533-40

18. Clemmensen P, Bates ER, Califf RM, et al. Complete atrioventricular block complicating inferior wall acute myocardial infarction treated with reperfusion therapy. TAMI Study Group. Am J Cardiol.1991 Feb 1;67(4):225-30

19. McCann P. A review of temporary cardiac pacing wires. Indian Pacing Electrophysiol J.2007 Jan 1;7(1):40-9.

20. Shah B, Awan ZA. Temporary pace makers implantation: do we need fluoroscopy? J Ayub Med Coll Abbottabad.2015 AprJun;27(2):284-6.

21. Metkus TS, Schulman SP, Marine JE, et al. Complications and Outcomes of Temporary Transvenous Pacing: An Analysis of > 360,000 Patients From the National Inpatient Sample. Chest.2019 Apr;155(4):749-757

22. Vanezis AP, Prasad R, Andrews R. Pacemaker leads and cardiac perforation. JRSM Open.2017 Feb 1;8(3):2054270416681432 